\title{
Le début d'une sclérose en plaques et vivre avec
}

\author{
Martine Pilloud-Meyer
}

Dr, FMH en psychiatrie et psychothérapie

Autour de mon $49^{\mathrm{e}}$ anniversaire, lors d'une course dans les Préalpes savoyardes le long d'une crête assez vertigineuse, je me servais beaucoup de mes deux mains pour me rassurer. Je me suis même surprise avec la question: "Pourquoi les êtres humains ont-ils abandonné la marche à quatre pattes? Ça serait tellement plus facile!» A ce moment, je me suis dit: «Il y a quelque chose qui ne tourne pas rond dans ta tête!» Peu avant, je m'étais trouvée deux fois par terre sans qu'il y ait eu un obstacle évident, en pleine ville. Depuis ce moment-là, j'ai commencé à me poser des questions sérieuses, étant donné que j'avais subi une névrite du nerf optique 14 ans plus tôt, à l'âge de 35 , une drôle de manifestation avec des douleurs à un œil et une vision brunâtre. A l'époque, les IRM n'existaient pas encore, et on m'avait conseillé de faire un examen par potentiel évoqué qui confirmait la névrite rétrobulbaire déjà soupçonnée. Nous avions appris à l'époque de notre final qu'en cas d'une névrite du nerf optique ni le patient, ni le médecin ne pouvait voir quelque chose, je n'avais donc pas d'emblée pensé à ce diagnostic. Notre petite fille de trois ans qui était propre depuis un certain temps, recommença à mouiller son lit... C'était donc évident que sa maman se faisait du souci, et j'ai décidé alors d'aller voir mon collègue neurologue.

Je suis psychiatre et j'avais à ce moment de ma vie un cabinet depuis une quinzaine d'années dans la même petite ville que lui. Mon collègue a l'âge de mon frère cadet et nous avons été mutuellement à l'inauguration de nos cabinets réciproque et partageons un bon nombre de patients. Il y a une relation agréable et amicale entre nous.

Mon collègue neurologue a été effrayé de me voir dans son cabinet avec une telle histoire, donc 15 ans après la première manifestation, mais la situation était assez claire. Je me suis demandé après-coup si c'était trop dur pour lui, que je l'avais consulté, mais en tout cas, il s'est toujours bien occupé de moi. J'ai dû consulter un autre neurologue pour obtenir un traitement à l'interféron ce qui fut à l'époque la seule option thérapeutique active envisageable. Les vrais problèmes moteurs sont seulement venus une dizaine d'années plus tard et je n'ai pas trop de soucis cognitifs. Chez ma sœur cadette de onze ans, la même maladie s'est déjà manifestée peu après ses vingt ans. En plus, je viens d'apprendre tout dernièrement que non seulement ma grand-mère paternelle, mais aussi sa mère, donc une de mes arrièregrand-mères, avait souffert vers la fin de sa vie d'une maladie neurologique bizarre qui l'empêchait de se déplacer normalement! Evidemment, le diagnostic de sclérose en plaques a seulement été posé pour ma sœur.

J'ai dû expérimenter sur ma propre personne combien les assurances n'apprécient guère les malades chroniques et essaient de beaucoup de manières de se libérer de leurs obligations. Pas seulement les séjours de réhabilitation, très utiles pour décharger les proches aidants, faire un bilan de la situation neurologique et de nouvelles propositions, mais aussi les deux séances de physiothérapie par semaine, absolument indispensables pour maintenir l'activité physique malgré la progression de la maladie, sont régulièrement refusés et mis en question. Il faut beaucoup de persévérance, un esprit combatif et du courage pour ne pas désespérer. Je me suis même posé la question, combien de malades ne sont pas trop malades pour réussir à continuer à se battre. L'attitude de beaucoup d'assurances est inhumaine et cruelle avec le seul but de gagner plus d'argent, plus du tout conforme à la raison pour laquelle elles ont été crées. Je me sens régulièrement profondément humiliée par l'attitude de mon assurance. Les maladies chroniques dont je m'occupe en tant que médecin sont les schizophrénies, les troubles bipolaires et anxieux. J'espère que ma maladie m'a au moins aidée à gagner plus de compréhension et d'empathie envers mes patients.

\section{Quelles expériences avez-vous faites?}

Vous est-il vous aussi arrivé, en tant que médecin, de vous retrouver tout à coup dans la peau d'un patient? Si votre histoire vous semble susceptible d'intéresser les lecteurs du BMS, n'hésitez pas à envoyer un article de 8000 signes maximum à l'adresse redaktion.saez[at]emh.ch - en cas d'évaluation positive par la rédaction, votre manuscrit sera publié dans le cadre de la série "Quand le médecin devient patient». 